\title{
Simposio \\ MODULACIÓN INMUNOLÓGICA Y TAENIA SOLIUM
}

\section{Inmunomodulación intestinal por helmintos: un tema de actualidad}

\author{
Ana Flisser \\ Departamento de Microbiología y Parasitología, Facultad de Medicina, \\ Universidad Nacional Autónoma de México, México, D.F., México
}

A partir de la segunda mitad del siglo $X X$ se ha presentado en el primer mundo un aumento impresionante de las enfermedades inmunológicas, entre ellas la enfermedad de Crohn y la colitis ulcerativa, que son enfermedades inflamatorias intestinales, así como asma, alergia a alimentos, rinitis alérgica, esclerosis múltiple y aun autismo.

Las enfermedades inflamatorias intestinales antes se presentaban en 1 de cada 5.000 a 10.000 personas y ahora se detectan en 1 de cada 200 a 500 individuos, especialmente en edades jóvenes. Este fenómeno se asocia con la hipótesis de la higiene, ya que la prevalencia de estas enfermedades aumenta en poblaciones que han adoptado prácticas higiénicas modernas, las que previenen la exposición a helmintos.

Las enfermedades inflamatorias intestinales son menos comunes en países tropicales en vías de desarrollo y los datos epidemiológicos sugieren que la gente portadora de helmintos tiene menos enfermedades inmunitarias. Por lo tanto, los helmintos intestinales -que son frecuentes en estos países-pueden prevenirenfermedadesinflamatorias intestinales y demás enfermedades inflamatorias mediante la inducción de circuitos reguladores de inmunidad protectora en las mucosas, e inhibir la inmunidad adaptativa asociada a la inflamación.

Se considera que los parásitos helmintos son expertos en modificar la actividad inmunológica de sus huéspedes, por lo que actualmente se ha generado gran interés en el estudio de los sistemas inmunitarios de los mamíferos en respuesta a sus parásitos metazoarios, utilizando modelos de roedores con diversas modificaciones inmunológicas, con la esperanza de identificar moléculas o mecanismos que se puedan traducir en mejores tratamientos para enfermedades autoinmunitarias e idiopáticas.

Hay múltiples datos que comprueban que la infección con helmintos puede reducir la gravedad de las enfermedades inflamatorias intestinales, por lo que se están desarrollando tratamientos basados en la administración de nematodos. Por ejemplo, Joel Weinstock trabaja con Trichuris suis, cuyos huevos constituyen ahora un fármaco aceptado por la Food and Drud Administration (FDA) para controlar el asma y otras entidades alérgicas, pues no contienen virus o bacterias, ni son mutagénicos. Además, en ensayos clínicos se ha demostrado que la exposición a helmintos reduce la colitis ulcerativa y la enfermedad de Crohn en pacientes que la padecen, debido a que se controla la inflamación.

Sus modelos en ratones con colitis, encefalitis, diabetes de tipo 1 o asma, que fueron infectados con el nemátodo Heligmosomoides polygyrus, se protegen de dichas enfermedades, lo que demuestra que las enfermedades inflamatorias intestinales se pueden prevenir o revertir. En este modelo se hizo evidente la importancia de la respuesta inmunitaria innata, por ejemplo, el hecho de que las células dendríticas sean parte crucial del proceso regulador de la respuesta inmunitaria $(1,2)$.

La inmunidad a los helmintos se caracteriza por una respuesta estereotípica asociada al desarrollo de linfocitos T cooperadores de tipo 2 (Th2), con presencia de eosinofilia, basofilia, niveles elevados de lgE y metaplasia celular del epitelio de la mucosa; además, aparecen macrófagos alternativamente activados en los tejidos afectados. Por ejemplo, la resistencia de los ratones a la infección con Trichuris muris se asocia a la presencia de eosinófilos en los ganglios linfáticos mesentéricos que drenan la mucosa, especialmente, durante las respuestas Th2 con producción de interleucina 4 (IL-4) (3).

La investigación de María Yazdanbakhsh y su grupo es muy interesante, pues midió las moléculas del sistema inmunitario en niños y adultos de Ghana y Gabón, comparando la de personas con 
esquistosomosis y las de individuos no parasitados; hay mayor producción de IL-4 en las personas parasitadas y la respuesta inmunitaria Th1 está, por lo general, suprimida. Por ejemplo, no se induce protección al vacunar contra influenza a niños de Gabón que tienen esquistosomosis, aunque hay producción de anticuerpos. Midieron células CD4+ en estos niños y vieron que después de la vacunación prácticamente no aumentaba IFN $\gamma$, TNFa ni IL2+, en comparación con los niños no infectados. También, estudiaron ligandos lipídicos de unión a receptores de tipo toll (Toll-like receptors) de esquistosomas y de bacterias, y demostraron que pueden generar distintos perfiles de citocinas dependiendo de su composición y, por lo tanto, generar diferentes respuestas inmunitarias; esta información es valiosa para identificar moléculas inmunomodulatorias $(4,5)$.

El grupo de David Artis ha sido clave en dilucidar la participación de los diferentes componentes inmunitarios a nivel intestinal en asociación con helmintos. Recientemente, demostró que un tipo celular de gran importancia son los basófilos, ya que llevan a cabo funciones esenciales en modelos de inmunidad y de inflamación dependientes de citocinas de tipo Th2, generadas después de la exposición a helmintos. Los basófilos migran a los ganglios linfáticos y presentan antígenos a linfocitos T CD4+, promoviendo su diferenciación hacia Th2, lo que pueden hacer en cooperación con células dendríticas. Además, los basófilos producen IL-4 e IL-13, citocinas que protegen contra helmintos intestinales al inducir a las células epiteliales a diferenciarse en células caliciformes que secretan la mucina RELM $\beta$, que es considerada como antihelmíntica (6).

Es interesante mencionar que se ha demostrado que las bacterias intestinales, que son comensales, pueden interactuar con los parásitos intestinales, ya que, por ejemplo, la disminución de las primeras reduce el número de huevos con embrión de T. muris; además, la supervivencia de este parásito depende de la microflora y coincide con la modulación de la respuesta inmunitaria del huésped, lo que apunta a interacciones críticas entre las bacterias (microflora) y los parásitos metazoarios (macrofauna), e introduce una dinámica nueva en el nicho intestinal, que tiene implicaciones fundamentales en los conceptos actuales de la homeostasis intestinal y la regulación de la inmunidad (7).

Rick Maizels, quien ha sido de los iniciadores en el estudio de la respuesta inmunitaria a helmintos, señala que debido a la evolución simultánea con el sistema inmunitario humano, estos organismos han aprendido a explotar las rutas inmunorreguladoras de sus huéspedes mamíferos, resultando, en muchos casos, en tolerancia asintomática de las infecciones. Cuando las infecciones y las respuestas inmunitarias resultantes no se regulan, generalmente se desarrollan enfermedades tanto agudas como crónicas (8).

Peter Hotez, primero en evaluar una vacuna contra la uncinarias (geohelmintos intestinales) en humanos, indica que los parásitos multicelulares eucariontes infectan a más de una cuarta parte de la población mundial, lo que causa un enorme gasto cuando se calculan los años de vida ajustados por incapacidad (disability-adjusted life year, DALY) (9):

$\begin{array}{lrrr}\text { Parasitosis por: } & \text { Infecciones } & \text { Muertes } & \text { DALY (en años) } \\ \text { Helmintos } & 1,3 \text { billones } & 415.000 & 114 \text { millones } \\ \text { Uncinarias } & 660 \text { millones } & 66.000 & 22 \text { millones } \\ \text { Esquistosomas } & 207 \text { millones } & 280.000 & 70 \text { millones }\end{array}$

Hay pocos estudios al respecto en cestodos. El modelo de Taenia solium adulta en hámsts, desarrollado en nuestro laboratorio, provee una buena herramienta para realizar estudios de la respuesta inmunitaria intestinal, los que se van a presentar durante este simposio (10).

\section{Referencias}

1. Elliott DE, Weinstock JV. Helminthic therapy: Using worms to treat immune-mediated disease. Adv Exp Med Biol. 2009;666:157-66.

2. Hang L, Setiawan T, Blum AM, Urban J, Stoyanoff K, Arihiro S, et al. Heligmosomoides polygyrus infection can inhibit colitis through direct interaction with innate immunity. J Immunol. 2010;185:3184-9.

3. Svensson M, Bell L, Little MC, DeSchoolmeester M, Locksley RM, Else KJ. Accumulation of eosinophils in intestine-draining mesenteric lymph nodes occurs after Trichuris muris infection. Parasite Immunol. 2011;33:1-11.

4. van Riet E, Adegnika AA, Retra K, Vieira R, Tielens $A G$, Lell B et al. Cellular and humoral responses to influenza in Gabonese children living in rural and semi-urban areas. J Infect Dis. 2007;196:1671-8.

5. Retra K, van Riet E, Adegnika AA, Everts B, van Geest S, Kremsner PG, et al. Immunologic activity of schistosomal and bacterial TLR2 ligands in Gabonese children. Parasite Immunol. 2008;30:39-46.

6. Siracusa MC, Comeau MR, Artis D. New insights into basophil biology: Initiators, regulators, and effectors of type 2 inflammation. Ann NY Acad Sci. 2011;1217:166-77.

7. Hayes KS, Bancroft AJ, Goldrick M, Portsmouth C, Roberts IS, Grencis RK. Exploitation of the intestinal 
microflora by the parasitic nematode Trichuris muris. Science. 2010;328:1391-4.

8. Maizels RM, Pearce EJ, Artis D, Yazdanbakhsh M, Wynn TA. Regulation of pathogenesis and immunity in helminth infections. J Exp Med. 2009;206:205966.

9. Hotez PJ, Bethony JM, Diemert DJ, Pearson M, Loukas A. Developing vaccines to combat hookworm infection and intestinal schistosomiasis. Nat Rev Microbiol. 2010;8:814-26.

10. Flisser A, Ávila G, Maravilla P, Mendlovic F, LeónCabrera S, Cruz-Rivera M, et al. Taenia solium: Current understanding of laboratory animal models of taeniosis. Parasitology. 2010;137:347-57.

\title{
Respuesta inmunitaria intestinal en el modelo experimental de teniasis
}

\author{
Guillermina Ávila \\ Departamento de Microbiología y Parasitología, Facultad de Medicina, \\ Universidad Nacional Autónoma de México, México, D.F., México
}

\section{Modelos experimentales de Taenia solium}

Taenia solium es un parásito que puede causar dos enfermedades en el ser humano, la cisticercosis y la teniasis, y en el cerdo, la cisticercosis. Mientras que la relación huésped-parásito en la cisticercosis ha sido estudiada ampliamente, en la teniasis es prácticamente desconocida, debido principalmente a que el ser humano es el único huésped definitivo natural.

Ante la problemática para profundizar en el conocimiento de la relación huésped-parásito de $T$. solium, los modelos experimentales ofrecen una alternativa para estudiar esta parasitosis. Taenia solium puede implantarse y crecer en diferentes roedores: hámster, gerbos y chinchillas. El desarrollo y recuperación de los parásitos son variables, únicamente se han obtenidos tenias con proglótidos grávidos y huevos infecciosos en chinchillas inmunodeprimidas con esteroides $(1,2)$.

El hámster presenta varias ventajas, ya que puede infectarse aun sin tratamiento con esteroides. El máximo desarrollo de las tenias es hasta proglótidos pregrávidos, por lo que no representan mayor riesgo para la salud del operador. Este modelo se utilizó inicialmente para desarrollar una prueba inmunodiagnóstica para teniasis mediante la captura de coproantígenos (3), para identificar la respuesta inmunitaria e humoral $(4,5)$, así como algunos componentes de la respuesta inflamatoria celular en la mucosa intestinal y, recientemente, para el ensayo de una vacuna (6).

Respuesta humoral en la teniasis experimental en el hámster

En hámsters infectados con el adulto de $T$. solium se estudió la cinética de la respuesta de anticuerpos lgG, tanto a nivel sistémico como intestinal, y se comparó con la presencia de antígenos de la tenia. Se tuvieron dos grupos de hámsters, uno de ellos se inmunodeprimió con acetato de metilprednisolona. Se tomaron muestras de heces y suero antes de la infección y, posteriormente, cada semana. Todos los hámsters desarrollaron anticuerpos $\operatorname{lgG}$ en suero, pero sólo se detectó lgG a nivel intestinal en heces de los animales no inmunodeprimidos.

En cuanto a la presencia de antígenos de $T$. solium, los animales fueron positivos en la ELISA para coproantígenos desde la primera semana de infección. Los hámsters no inmunodeprimidos expulsaron los parásitos más pronto y hubo animales positivos a las 11 semanas de la infección, en comparación con los animales tratados con metilprednisolona que permanecieron infectados hasta las 17 semanas.

Por el contrario, sólo se detectaron antígenos de la tenia en circulación en los hámsters no inmunodeprimidos a partir de la tercera semana de la infección y hasta una semana antes de que la tenia fuera eliminada. Probablemente, los antígenos de la tenia pueden cruzar la mucosa intestinal y alcanzar la circulación debido a la inflamación local cuando las respuestas inmunitaria e inflamatoria están intactas, pero cuando son inmunodeprimidos con esteroides, se bloquean ambas respuestas y, por consiguiente, el parásito puede sobrevivir por más tiempo (5).

Respuesta celular en la mucosa intestinal de gerbos y hámsters infectados con Taenia solium

Se estudió la respuesta inflamatoria desarrollada en la mucosa intestinal de gerbos y hámsters infectados con el adulto de $T$. solium y no inmunodeprimidos. Los roedores se sacrificaron a diferentes días después de la infección, se obtuvo el intestino delgado y se abrió longitudinalmente para localizar las tenias, contarlas y medirlas; también, se tomó una biopsia de la mucosa 
intestinal que rodeaba los escólices, se fijó y procesó histológicamente para la obtención de cortes que fueron teñidos con hematoxilina-eosina, ácido peryódico de Schiff (PAS), Giemsa o Wright y se observaron al microscopio.

El aspecto de la mucosa intestinal en los animales no infectados era normal, con vellosidades delgadas, alargadas y redondeadas, con algunas células caliciformes en el epitelio intestinal, escasos eosinófilos, linfocitos, células plasmáticas y cebadas esparcidas a lo largo de la lámina propia. En los animales infectados, el aspecto normal de la mucosa se perdió, las vellosidades estaban acortadas y deformes, y se presentó edema.

La reacción inflamatoria se observó en la mucosa intestinal que rodeaba los escólices, hubo un ligero aumento de células plasmáticas, linfocitos, fibroblastos, eosinófilos y macrófagos, y sólo las células caliciformes y las cebadas aumentaron de forma considerable. Las células caliciformes se incrementaron hasta cinco veces en hámsters y ocho en los gerbos; el aumento se presentó a los 13 días de la infección en gerbos y a los 18, en hámsters; durante la primera semana de infección, las células caliciformes se observaron redondas y pequeñas; posteriormente, aumentaron de tamaño y hubo hipersecreción de moco.

En cuanto a las células cebadas, éstas aumentaron sólo en los gerbos; el incremento fue ocho veces mayor en comparación con los controles no infectados y el pico se registró a los 19 después de la infección, al mismo tiempo que se aumentó la concentración de histamina en el contenido intestinal. La recuperación de tenias estuvo inversamente relacionada con el número de células caliciformes y cebadas, las que finalmente disminuyeron cuando las tenias fueron expulsadas.

Los gerbos que desarrollaron una respuesta inflamatoria mucho más intensa fueron menos permisivos a la infección por $T$. solium, las tenias recuperadas midieron de 1 a $2 \mathrm{~cm}$ y los animales dejaron de estar infectados a los 25 , mientras que los ejemplares obtenidos de los hámsters llegaron a medir hasta $40 \mathrm{~cm}$ y algunos animales mantuvieron la infección por 46 días (4).

Posteriormente, se estudió el perfil de las citocinas de los linfocitos Th en la mucosa intestinal de los hámsters. Se buscó la expresión de ARNm para IFN- $\gamma$, IL-4, IL-5 e IL-13 a diferentes tiempos de la infección. Se diseñaron sondas antisentido de ADNc para cada una de las citocinas según las secuencias reportadas en el GenBank, se hizo el análisis in silico para verificar su especificidad y, posteriormente, se marcaron con digoxigenina para emplearse en ensayos de hibridación in situ. Los hámsters infectados mostraron reacción positiva para el ARNm de IFN- $\gamma$ e IL-13 a los 2 y 4 días después de la infección, mientras que la IL-4 se detectó a los 6 días y la IL-5 hasta los 8 días. El número de animales positivos para cada citocina se incrementó con el tiempo de infección, excepto para el IFN- $\gamma$ a los 16 después de la infección, cuando comenzó a disminuir el número de hámsters positivos y hubo predominancia del ARNm para citocinas de tipo Th2. En los hámsters no infectados no hubo reacción positiva para las diferentes citocinas.

Estos resultados demuestran que $T$. soliuminduce una respuesta mezclada Th1/Th2 en la mucosa intestinal de los hámsters, con predominancia de una respuesta Th2 después de dos semanas de la infección, cuando las tenias comienzan a ser eliminadas (7).

\section{Referencias}

1. Verster A. Preliminary report on the golden hamster as a definitive host of Taenia solium Linnaeus, 1758 and Taenia saginata Goeze, 1782. Onderstepoort J Vet Res. 1971;38:63-4.

2. Maravilla P, Ávila G, Cabrera V, Aguilar L, Flisser A. Comparative development of Taenia solium in experimental models. J Parasitol. 1998;84:882-6.

3. Allan JC, Ávila G, García J, Flisser A, Craig PS. Immunodiagnosis of taeniasis by coproantigen detection. Parasitology. 1990;101:473-7.

4. Ávila G, Aguilar L, Benítez S, Yepez-Mulia L, Lavenat I, Flisser A. Inflammatory responses in the intestinal mucosa of gerbils and hamsters experimentally infected with the adult stage of Taenia solium. Int $\mathrm{J}$ Parasitol. 2002;32:1301-8.

5. Ávila G, Benítez M, Aguilar-Vega L, Flisser A. Kinetics of Taenia solium antibodies and antigens in experimental taeniosis. Parasitol Res. 2003;89:284-9.

6. León-Cabrera S, Cruz-Rivera M, Mendlovic F, Ávila-Ramírez G, Carrero JC, Laclette JP, et al. Standardization of an experimental model of human taeniosis for oral vaccination. Methods. 2009;49:34650.

7. Ávila G, Aguilar L, Romero-Valdovinos M, GarcíaVázquez F, Flisser A. Cytokine response in the intestinal mucosa of hamsters infected with Taenia solium. Ann NY Acad Sci. 2008;1149:170-3. 


\title{
Detección de citocinas en tiempo real en el modelo experimental de teniasis
}

\author{
Mayra Cruz-Rivera \\ Departamento de Microbiología y Parasitología, Facultad de Medicina, \\ Universidad Nacional Autónoma de México, México, D.F., México
}

Taenia solium es un parásito helminto que causa dos enfermedades en el ser humano: la cisticercosis y la teniasis. La neurocisticercosis es una enfermedad importante de salud pública que se produce por el establecimiento del metacestodo de $T$. solium en el sistema nervioso central (1). El adulto se establece en el intestino del ser humano a consecuencia de consumir carne de cerdo que contiene cisticercos y está mal cocinada lo que permite que se desarrolle la tenia $(2,3)$.

Debido a que el ser humano es el único huésped definitivo natural para $T$. solium, se desconocen muchos aspectos de la relación huésped-parásito; por esto, se han desarrollado varios modelos experimentales en los que se pueden llevar a cabo diversos estudios, y el hámster es el modelo más propenso a la infección $(4,5)$.

Por hibridación in situ, se ha encontrado que durante la infección se producen citocinas de tipo Th1 y Th2 durante las dos primeras semanas de la infección y después existe un cambio hacia una respuesta Th2. A los 2 días de la infección, las citocinas que se detectan son IFN- $\gamma$ e IL-13; a los 6 y 8 días, IL-4 e IL-5, respectivamente, y a los 16 días, todos los hámsters son positivos para IL-13 e IL-5, y sólo el $20 \%$ para IFN- $\gamma(6)$.

Tomando en cuenta que la respuesta celular local del huésped ante el establecimiento del parásito en el intestino no ha sido completamente caracterizada, proponemos el desarrollo de PCR en tiempo real para la detección específica de ARN mensajero de citocinas a nivel local en el sitio de anclaje de la tenia. El perfil de citocinas y otros mediadores producidos por las células presentes en el infiltrado cercano a la zona de anclaje, puede jugar un papel importante tanto en la tolerancia a la infección como en su eliminación.

Nuestro objetivo fue determinar el perfil de citocinas en la teniasis experimental, mediante la cuantificación relativa de la expresión de INF- $\gamma$, IL-4, IL-10, IL-12, IL-13 y FOX P3, a lo largo de la infección, mediante PCR en tiempo real.

Se diseñaron oligonucleótidos para diversas interleucinas de tipo Th1 y Th2. Se llevó a cabo la extracción de ARN total del tejido de la zona de anclaje de los intestinos obtenidos de hámsters a diferentes tiempos después de la infección: $3,7,10$,
20 y 30 días. Se procedió con la síntesis de ADNc mediante la reacción de transcriptasa inversa. El PCR se realizó utilizando el ADNc proveniente de 5 ug de ARN y el uso de una mezcla de reacción comercial (Syber green). Todas las muestras se analizaron en el Light Cycler 480® Roche. Después de la amplificación, se determinó la presencia de los amplicones correspondientes, mediante el análisis de las curvas de disociación (Melting Curve Analysis), para definir la temperatura de disociación de cada fragmento. Finalmente, se llevó a cabo la cuantificación relativa de cada citocina con respecto al gen constitutivo (HPRT).

EI PCR en tiempo real provee una herramienta sensible, útil y práctica para la determinación cuantitativa de citocinas de hámster a nivel de su expresión, ya que no se cuenta con reactivos específicos para su identificación a nivel de proteínas.

Esta metodología será de gran utilidad para estudiar la relación huésped-parásito y la respuesta inmunitaria que ocurre en el tubo digestivo. Más aún, nos permitirá entender los mecanismos inmunológicos por los cuales la tenia es expulsada del huésped.

\section{Referencias}

1. Flisser A, Rodríguez-Canul R, Willingham AL. Control of the taeniosis/cysticercosis complex: Future developments. Vet Parasitol. 2006;139:283-92.

2. Flisser A. Taeniasis and cysticercosis due to Taenia solium. In: Tsieh Sun, editor. Progress in clinical parasitology. Boca Raton: CRC Press Inc.; 1994. p. 77-116.

3. Schantz PM. Taenia solium cysticercosis: An overview of global distribution and transmission. In: Singh G, Prabhakar S, editors. Taenia solium cysticercosis: From basic to clinical science. Oxon, UK.: CABI Publ.; 2002. p. 63-73.

4. Maravilla P, Ávila G, Cabrera V, Aguilar L, Flisser A. Comparative development of Taenia solium in experimental models. J Parasitol. 1998;84:882-6.

5. Ávila G, Teran N, Aguilar-Vega L, Maravilla $P$, Mata-Miranda P, Flisser A. Laboratory animal models for human Taenia solium. Parasitol Int. 2006;55(Suppl.):S99-103.

6. Ávila G, Aguilar L, Romero-Valdovinos M, GarcíaVázquez F, Flisser A. Cytokine response in the intestinal mucosa of hamsters infected with Taenia solium. Ann NY Acad Sci. 2008;1149:170-3. 


\title{
Respuesta immunitaria contra calreticulina de Taenia solium
}

\author{
Fela Mendlovic ${ }^{1,2}$ \\ 1 Facultad de Medicina, Universidad Nacional Autónoma de México, México, D.F., México \\ 2 Facultad de Ciencias de las Salud, Universidad Anáhuac del Norte, Huixquilucan, México
}

Taenia solium causa dos enfermedades importantes en el ser humano. La mayor parte de la investigación se ha enfocado en el estadio larvario o metacestodo, debido a que causa neurocisticercosis, una de las parasitosis más comunes del sistema nervioso central. El portador del parásito adulto es el principal factor de riesgo para adquirir neurocisticercosis y, sin embargo, se conoce muy poco sobre la inmunidad intestinal inducida durante la teniasis. Esto se debe a que el ser humano es el único huésped definitivo de la tenia y, por lo tanto, es necesario recurrir a modelos experimentales para estudiarla. Desde hace varios años, en el laboratorio hemos utilizado el modelo experimental del hámster dorado para determinar los parámetros inmunológicos y de desarrollo de $T$. solium $(1,2)$.

La calreticulina es una proteína ubicua y muy conservada, involucrada en la homeostasis intracelular de $\mathrm{Ca}^{2+}$ y en el plegamiento correcto de proteínas "glucosiladas" (glycosylated). Estas dos funciones denotan su importancia e influencia en diversos procesos celulares (3). Inicialmente, fue descrita en la luz del retículo endoplásmico y, recientemente, se ha identificado en la superficie de varias células, así como en productos de excreción y secreción de varios helmintos $(4,5)$. La calreticulina interactúa con el sistema inmunitario del huésped, pues los pacientes con tripanosomosis, esquistosomosis y oncocercosis tienen anticuerpos anticalreticulina $(6,7)$ e, interesantemente, se expresa exageradamente en las parasitosis intestinales crónicas en el ratón (8). Además, se ha demostrado que la calreticulina de Heligmosomoides polygyrus se secreta, induce la producción de IL-4 e IL-10, y se une a células dendríticas por medio del receptor carroñero $A(9)$. Se ha propuesto que la calreticulina de parásitos podría representar un patrón molecular asociado a patógenos capaz de interactuar con células dendríticas y dirigir la respuesta inmunitaria hacia un perfil Th2 $(10,11)$.

En nuestro laboratorio, identificamos y expresamos de forma recombinante la calreticulina de $T$. solium, la que se expresa de manera diferencial durante la gametogénesis y la embriogénesis, además de estar presente en los miocitones de las ventosas y en los citones subtegumentarias; ambos tipos celulares se encuentran en estrecho contacto con la mucosa intestinal del huésped $(12,13)$. La calreticulina de $T$. solium está presente en los productos de excreción y secreción de tenias cultivadas in vitro. Además, hemos demostrado que en ratones inmunizados por vía oral con la calreticulina de $T$. solium, se induce la producción de citocinas de tipo Th2 en los ganglios linfáticos mesentéricos e IgA específica, tanto a nivel local como sistémico. La administración subcutánea de esta proteína induce preferentemente anticuerpos IgG1 asociados también a respuestas de tipo 2 (14).

En el modelo experimental de la teniasis, la calreticulina de $T$. solium induce proliferación de linfocitos de bazo de animales a los 20 días después de la infestación, mientras que los linfocitos de ganglios linfáticos mesentéricos no responden a este estímulo. Al analizar la respuesta a un sobrenadante de tenias después de ser sometidas a ultrasonido (sonicated), se observa un patrón similar, con una mayor estimulación de las células del bazo. En cuanto a la respuesta humoral, 100 y $50 \%$ de los animales presentan anticuerpos séricos específicos contra el el sobrenadante y la calreticulina de $T$. solium, respectivamente.

Nuestros datos sugieren que durante la infección por el parásito adulto de $T$. solium, hay una modulación de la respuesta inmunitaria en las mucosas. Además, dicha calreticulina induce preferentemente una respuesta de tipo Th2, que es una cualidad que caracteriza a los helmintos, y crea un ambiente antiinflamatorio que podría ser explotado con fines terapéuticos de inmunomodulación.

\section{Referencias}

1. Maravilla P, Ávila G, Cabrera V, Aguilar L, Flisser A. Comparative development of Taenia solium in experimental models. J Parasitol. 1998;84:882-6.

2. Merchant MT, Aguilar L, Ávila G, Robert L, Flisser A, Willms K. Taenia solium: Description of the intestinal implantation sites in experimental hamster infections. J Parasitol. 1998;84:681-5.

3. Michalak M, Corbett EF, Mesaeli N, Nakamura K, Opas M. Calreticulin: One protein, one gene, many functions. Biochem J. 1999;344:281-92.

4. Suchitra S, Joshi P. Characterization of Haemonchus contortus calreticulin suggests its role in feeding and 
immune evasion by the parasite. Biochim Biophys Acta. 2005;1722:293-303.

5. Guillou F, Roger E, Mone Y, Rognon A, Grunau $\mathrm{C}$, Theron $\mathrm{A}$, et al. Excretory-secretory proteome of larval Schistosoma mansoni and Echinostoma caproni, two parasites of Biomphalaria glabrata. Mol Biochem Parasitol. 2007;155:45-56.

6. Rokeach LA, Zimmerman PA, Unnasch TR. Epitopes of the Onchocerca volvulus RAL1 antigen, a member of the calreticulin family of proteins, recognized by sera from patients with onchocerciasis. Infect Immun. 1994;62:3696-704.

7. Marcelain K, Colombo A, Molina MC, Ferreira L, Lorca M, Aguillon JC, et al. Development of an immunoenzymatic assay for the detection of human antibodies against Trypanosoma cruzicalreticulin, an immunodominant antigen. Acta Trop. 2000;75:291300.

8. Morgan C, LaCourse EJ, Rushbrook BJ, Greetham D, Hamilton JV, Barrett J, et al. Plasticity demonstrated in the proteome of a parasitic nematode within the intestine of different host strains. Proteomics. 2006;6:4633-45.

9. Rzepecka J, Rausch S, Klotz C, Schnoller C, Kornprobst T, Hagen J, et al. Calreticulin from the intestinal nematode Heligmosomoides polygyrus is a Th2-skewing protein and interacts with murine scavenger receptor-A. Mol Immunol. 2009;46:110919.

10. Mendlovic F, Flisser A. Dendritic cells in the gut: Interaction with intestinal helminths. J Biomed Biotechnol. 2010:250563.

11. Terrazas CA, Terrazas LI, Gómez-García L. Modulation of dendritic cell responses by parasites: A common strategy to survive. J Biomed Biotechnol. 2010:357106.

12. Mendlovic F, Carrillo-Farga J, Torres J, Laclette JP, Flisser A. Differential expression of calreticulin in developmental stages of Taenia solium. J Parasitol. 2006;92:789-95.

13. Mendlovic F, Ostoa-Saloma P, Solís CF, MartínezOcana J, Flisser A, Laclette JP. Cloning, characterization, and functional expression of Taenia solium calreticulin. J Parasitol. 2004;90:891-3.

14. Fonseca-Coronado S, Ruiz-Tovar K, Pérez-Tapia M, Mendlovic F, Flisser A. Taenia solium: Immune response against oral or systemic immunization with purified recombinant calreticulin in mice. Exp Parasitol. 2010;127:313-7.

\title{
Respuesta protectora inducida por la calreticulina de Taenia solium
}

\author{
Sonia León-Cabrera \\ Departamento de Microbiología y Parasitología, Facultad de Medicina, \\ Universidad Nacional Autónoma de México, México, D.F., México
}

\section{Introducción}

La neurocisticercosis humana es causada por el estado larvario del cestodo Taenia solium. Los estudios epidemiológicos han demostrado que el portador de la tenia adulta intestinal en el hogar es el principal factor de riesgo para adquirir cisticercosis (1). La vacunación es una alternativa para prevenir el establecimiento o el desarrollo del parásito adulto. Debido a que la infección ocurre por vía oral, es necesario inducir una efectiva y eficiente inmunidad intestinal. La calreticulina de Taenia solium es una proteína multifuncional presente en las células subtegumentarias y musculares del cisticerco y la tenia; además, se expresa de manera diferencial durante la gametogénesis y la embriogénesis (2). Esta proteína es reconocida por su capacidad de inducir respuesta inmunitaria de tipo 2 (3), determinante para la protección contra helmintos intestinales.

\section{Objetivo}

Evaluar la protección inducida por la vacunación oral con calreticulina de Taenia solium en el modelo experimental de teniasis intestinal en el hámster dorado.

\section{Materiales y métodos}

Se obtuvo calreticulina de Taenia solium en un sistema de expresión bacteriano y con esta proteína más toxina colérica como adyuvante, se inmunizaron hámsters por vía oral en cuatro ocasiones una vez por semana. Los grupos control fueron inmunizados con toxina colérica o con el diluyente. Quince días después de la última inmunización, los hámsters se retaron por vía oral con cuatro cisticercos provenientes de un cerdo infectado de manera natural. Durante la necropsia se determinó el porcentaje de infección, y la longitud y la localización de los parásitos en el intestino. La respuesta inmunitaria se evaluó por medio de ELISA, cortes histológicos, proliferación linfoide y PCR en tiempo real.

\section{Resultados}

La inmunización oral con calreticulina de Taenia solium indujo una reducción en el número de tenias de $44 \%$ hasta $100 \%$, dependiendo de las 
características de los hámsters y de los cisticercos utilizados (4). En los animales inmunizados, las tenias encontradas fueron pequeñas y modificaron su zona de anclaje en el intestino. El análisis de la respuesta inmunitaria mostró un incremento en la producción de anticuerpos IgA en la mucosa e IgG sistémico, después de la segunda inmunización. La inmunización oral con calreticulina de Taenia solium provocó un incremento en el número de células caliciformes y un aumento en la expresión de IL-4 en la zona de anclaje de los parásitos en el intestino.

\section{Conclusiones}

La vacunación con calreticulina de Taenia solium induce una respuesta inmunitaria protectora contra el estadio adulto de T. solium; por lo tanto, es un candidato interesante para el desarrollo de una vacuna oral de uso humano.

\section{Referencias}

1. Flisser A. Risk factors and control measures for taeniosis/cysticercosis. In: Craig P, Pawlowski Z, editors.Cestode zoonoses: Echinococcosis and cysticercosis, an emergent and global problem. NATO Science Series. Amsterdam: IOS Press; 2002. p. 335-42.

2. Mendlovic F, Carrillo-Farga J, Torres J, Laclette JP, Flisser A. Differential expression of calreticulin in developmental stages of Taenia solium. J Parasitol. 2006;92:789-95.

3. Rzepecka J, Rausch S, Klotz C, Schnoller C, Kornprobst $\mathrm{T}$, Hagen $\mathrm{J}$, et al. Calreticulin from the intestinal nematode Heligmosomoides polygyrus is a Th2skewing protein and interacts with murine scavenger receptor-A. Mol Immunol. 2009;46:1109-19.

4. León-Cabrera $S$, Cruz-Rivera $M$, Mendlovic $F$, Ávila-Ramírez G, Carrero JC, Laclette JP, et al. Standardization of an experimental model of human taeniosis for oral vaccination. Methods. 2009;49:34650. 


\author{
Simposio \\ Nuevas alternativas terapéUticas \\ PARA EL MANEJO DE LA MALARIA
}

\title{
Situación de la malaria a nivel mundial y la contribución de Sanofi a través de su programa de acceso a medicamentos
}

\author{
Robert Sebbag \\ Sanofi, Gentilly, Francia
}

La documentación histórica de la malaria se remonta a más de 4.000 años. Los síntomas de la malaria fueron descritos en antiguos manuscritos médicos chinos. En el año 2700 a. C., varios síntomas característicos de lo que más tarde recibiría el nombre de malaria, fueron consignados en el Nei Ching, el canon de la Medicina (1).

Han pasado varios siglos y la malaria sigue siendo un motivo de preocupación en nuestro planeta. La mitad de la población mundial vive hoy en áreas de riesgo de transmisión de la malaria en 109 países y territorios (2). En 2008 se informaron más de 200 millones de casos, que causaron la muerte a 800.000 personas, principalmente en áreas tropicales del África subsahariana. Se trata de la quinta causa de muerte por enfermedades infecciosas en el mundo entero (2). La malaria también tiene importantes efectos económicos, ya que perpetúa el círculo vicioso de la pobreza al desacelerar el crecimiento económico y el desarrollo de los pueblos.

Una parte de la historia de la malaria corresponde a un extenso capítulo de éxitos y fracasos terapéuticos con diversos medicamentos que fueron presentados en el momento de su descubrimiento como la solución milagrosa y que pocos años más tarde se vio que no lograron cumplir las expectativas.

En la primera década del siglo XXI, la Organización Mundial de la Salud (OMS) decidió cambiar los lineamientos del tratamiento del paludismo por Plasmodium falciparum, mediante la introducción de la terapia combinada basada en derivados de la artemisinina, como una estrategia para retrasar la aparición de la resistencia.

Entre tanto, se produjo una movilización política mundial para combatir la malaria que se tradujo en el compromiso de los presidentes africanos con la declaración de Abuja, el nacimiento de varias iniciativas de la comunidad internacional y la aparición en la escena de Roll Back Malaria y el Fondo Global de Lucha contra el Sida, la Tuberculosis y la Malaria, que promovieron la creación de alianzas de todos los orígenes con el objeto de unificar esfuerzos y hacerle frente a la enfermedad, pero, más importante aún, trajeron a escena una parte considerable de los recursos financieros faltantes necesarios para alcanzar resultados efectivos.

En concordancia con esta movilización internacional, y teniendo en cuenta que los medicamentos por sí solos no son suficientes, Sanofi creó el programa Impact Malaria en su Departamento de Acceso a Medicamentos para acompañar a los productos en el campo. La malaria no va a ser eliminada solo con medicamentos. Existe la necesidad de que los pacientes y la población expuesta a la enfermedad la entiendan mejor para poder prevenirla. Para tal efecto, Impact Malaria ha desarrollado varias herramientas educativas dirigidas a la población, a niños y adultos, tomando en consideración su ambiente y sus particularidades. Estas herramientas se encuentran a disposición de los países y sus programas nacionales y se pueden adaptar al contexto local.

Asimismo, las buenas prácticas clínicas constituyen una prioridad en la malaria, si existe la expectativa real de erradicar la enfermedad. Se han desarrollado varios programas de entrenamiento y actividades de construcción de destrezas para ayudar a mejorar las capacidades de los profesionales de la salud, en asociación con programas nacionales y locales de control de la malaria e institutos de enseñanza, investigación o entrenamiento; hasta la fecha, cerca de 500 personas han recibido entrenamiento gracias a Sanofi a través de diferentes alianzas.

En paralelo, teniendo en cuenta sus 70 años de experiencia en el campo de la malaria, Sanofi establecióunaalianzaconDNDi(DrugsforNeglected 